\title{
Evaluación psicopedagógica en el contexto de atención a la diversidad
}

\section{Psychopedagogic assessment in the contexto of care for diversity}

\author{
Jorge Ivan Correa Alzate ${ }^{1}$
}

\section{Resumen}

Para dar respuesta educativa a todos los estudiantes en el marco de la atención a la diversidad, surge la psicopedagogía, como opción para apoyar y orientar la acción educativa, posibilitando criterios de evaluación e intervención en el diseño, desarrollo, innovación y valoración de los procesos educativos.

La Psicopedagogía, en su papel de colaborativa a los procesos de aprendizaje en la educación, define formas para conocer, registrar y analizar lo que sucede en la interacción de los estudiantes en diferentes contextos, valora los diferentes elementos que surgen en el proceso de aprendizaje de los estudiantes, presenta herramientas que posibilitan identificar la forma como se manifiestan las capacidades de los estudiantes con las características de los contextos, reconoce las condiciones que brindan los contextos para el aprendizaje y concibe el aprendizaje como fruto de la interacción social.

El texto aborda el papel de la evaluación psicopedagógica como estrategia para orientar las adaptaciones en el currículo y no la adaptación de la evaluación, señalando la relación directa entre evaluación y aprendizaje en el proceso formativo del estudiante.

Palabras claves: diversidad, psicopedagogía, flexibilidad curricular, técnicas

Docente Titular. Líder del grupo de investigación SENDEROS Facultad de Educación del Tecnológico de Antioquia. Director del programa de Educación Inclusiva, Convenio Ministerio de Educación Nacional - Tecnológico de Antioquia. 
Talea Jorge Ivan Correa Alrate

\begin{abstract}
Intending to provide a pedagogic response to all students concerning care for diversity, psychopedagogy arose as a way to support and orientate educative action, allowing for assessment criteria and intervening in design, development, innovation and assessment of educational processes.
\end{abstract}

Psychopedagogy, according to its role as a cooperative tool in educational learning processes, defines several ways to learn, register and analyse what happens with student interaction in varied contexts, estimates the various elements arising in student learning processes, presents tools to identify the way students' skills show themselves among context features, acknowledges conditions for learning provided by contexts and thinks of learning as a result from social interaction.

This paper addresses the role of psychopedagogic assessment as a strategy to guide adjustments to curriculum rather than adjusting evaluation, which points to a direct relation between evaluation and learning in the students' formation process.

Keywords: diversity, psychopedagogy, curricular flexibility, techniques.

Para dar respuesta educativa a todos los estudiantes en el marco de la diversidad, la psicopedagogía, surge para apoyar y orientar la acción educativa, posibilitando criterios de evaluación e intervención en el diseño, desarrollo, innovación y evaluación de los procesos educativos. La psicopedagogía se constituye en un puente de unión entre la teoría explicativa y la acción práctica y está presente en la Relación con las prácticas educativas escolares (Correa, 2001); Disponiendo de los servicios de apoyo, en áreas que soportan la atención como la Sicología, la fonoaudiología y trabajo social entre otros; de estrategias para canalizar las necesidades de aprendizaje de la población en su diversidad, en función del contexto de desempeño cotidiano del estudiante; de la inclusión de materiales técnicos y didácticos, que son inmersos como adecuaciones en el proceso de la orientación pedagógica; de la formación del profesorado para facilitar la compresión de las necesidades educativas de cada estudiante y de la investigación educativa como herramienta para dar cuenta de la evolución de procesos pedagógicos.
La psicopedagogía en la atención a la diversidad, reconoce el concepto de necesidad educativa, bajo la mirada de interactiva, es decir como una situación que emerge y no como una condición personal, ni estática en la persona; no se analiza como inherente al estudiante, sino que se visualiza como la dificultad, la brecha o el obstáculo que surge en el momento, en el que el niño, la niña, o el joven interactúan en el contexto escolar, dando como clave fundamental, que la orientación se brinde desde el contexto mismo, bajo postulados propios de la pedagogía.

La Psicopedagogía, en su papel de colaborativa a los procesos de aprendizaje de los estudiantes, define formas para conocer, registrar y analizar lo que sucede en la interacción de ellos en diferentes contextos; en el campo educativo, valora los diferentes elementos que surgen en el proceso de aprendizaje, identificando la forma como interactúan sus capacidades con las características de los contextos; además reconoce las condiciones que brindan éstos para el aprendizaje; identificándose con acciones 
de carácter preventivo en el acompańamiento pedagógico (estrategia de apoyo para los estudiantes que requieren orientaciones especificas, en función acceder al aprendizaje).

El acompañamiento pedagógico, se aplica en el contexto de ambientes de aprendizaje, entendidos como el conjunto de propiedades organizativas tanto instructivas como Psicosociales, que permiten describir la vida de los espacios pedagógicos, y de los entornos institucional y social, muy cercanos a los utilizados por profesores y estudiantes; constituyéndose en el escenario por excelencia de la enseñanza. Desde una perspectiva ambiental o socioecológica se estudian las interacciones que docentes y estudiantes registran sobre los elementos académicos (metodologías, materiales), motivacionales (atención, implicación, persistencia), conductuales (normas de convivencia), afectivos e interpersonales (apoyo emocional, cordialidad), del aula. De acuerdo con esto, la enseñanza y el aprendizaje se entienden como procesos continuos de intercambio cognitivo y social, en los cuales se detectan factores que pueden interpretarse, propiamente, como del contexto. De esta forma, es posible abordar las estrategias en el currículo como algo más que herramientas meramente individuales o de creación exclusivamente por la influencia de los factores personales del profesor, significa que el acompańamiento se orienta de manera holística, comprendiendo la complejidad del contexto y de la totalidad de recursos y situaciones, dentro de los que se sitúa el aprendizaje que le proporciona el contexto a los estudiantes.

En el momento que se transforma la educación Especial como el conjunto de recursos y medidas que se ponen en juego para responder a las Necesidades Educativas de los estudiantes, toma fuerza el aporte de la psicopedagogía en el campo de la educación formal, con tres tendencias básicas:

a) Lograr el mayor grado de desarrollo de las capacidades para todos y cada uno de los estudiantes, incluidos aquellos cuyas necesidades educativas se han descrito como individuales.

b) Orientar modelos pedagógicos que respondan al desarrollo de las competencias básicas, ciudadanas y laborales, realizando las adaptaciones a la didáctica para dar respuesta a la formación de la población en general.

c) Orientar la práctica pedagógica bajo esquemas de flexibilidad e innovación con relación a la planeación e incorporación de los servicios de apoyos.

Además la psicopedagogía aporta modelos, enfoques, métodos y estrategias que permitan la materialización metodológica de las tendencias descriptas:

Modelo preventivo, considera el nivel de desarrollo del estudiante, la evaluación, se organiza para detectar intereses y motivaciones, buscando maximizar el potencial de la persona y a nivel del currículo se está atento a prevenir las situaciones que puedan generar fracaso en el logro del aprendizaje.

Modelo comunitario, la atención educativa y la promoción del ser humano es un compromiso de todos; la evaluación se interesa en analizar los factores de desempeño social y el currículo trabaja en torno al aprendizaje cooperativo y la mediación social de pares, adultos y personas con el reconocimiento propio del contexto.

Enfoque constructivista, muestra interés por la construcción del conocimiento y las 
estructuras cognitivas que intervienen en el aprendizaje, la evaluación se centra en los esquemas para la aprehensión del conocimiento y el currículo se orienta al desarrollo de habilidades de pensamiento.

Enfoquehumanista, valoralaautodeterminación en el ser humano, fomenta la autoevaluación y la orientación desde la persuasión, el currículo trabaja en torno a estrategias para la autorregulación.

Enfoque ecológico, la educación es de tipo intercultural, interés a nivel de la evaluación en conocer la valoración por la diferencia y el currículo privilegia la comunicación de procesos, necesidades y la orientación de estrategias para el cambio de actitudes que generan discriminación.

La (UNESCO, 2004) recomienda la evaluación como parte de una educación de calidad, bajo los siguientes objetivos:

* El objetivo de la evaluación es hacer posible que los docentes e instituciones educativas den respuesta a una amplia diversidad de estudiantes. La evaluación ha de ayudar a los maestros a planificar para atender la diversidad en sus aulas y ayudar al desarrollo de las escuelas para que sean más inclusivas.

* La mayoría de las evaluaciones de mayor utilidad son llevadas a cabo por los mismos docentes y es necesario poner a su disposición un amplio rango de técnicas a través de la formación y actualización permanente.

* Cuándo la evaluación es realizada por un especialista, ésta debe proporcionar información para la toma de decisiones educativas respecto a cómo debe enseñarse a los estudiantes. Esto será más fácil si los especialistas interactúan en las instituciones mismas y si trabajan en estrecha colaboración con los docentes.
* Los padres, las familias y los estudiantes pueden hacer contribuciones importantes al proceso de evaluación.

* Una evaluación temprana de las dificultades emergentes es esencial para llevar a cabo un acompañamiento oportuno. Una evaluación temprana no se refiere sólo a los primeros años de la vida del niño (a), sino también a la identificación de posibles problemas en cualquier ciclo vital del desarrollo humano.

La evaluación en el Proyecto Educativo Institucional, según el Ministerio de Educación Nacional, 2009, artículos 3) tiene los siguientes propósitos:

- Identificar las características personales, intereses, ritmos de desarrollo y estilos de aprendizaje del estudiante para valorar sus avances (...) en los estudiantes.

- Proporcionar información básica para consolidar o reorientar los procesos educativos relacionados con el desarrollo integral del estudiante.

- Suministrar información que permita implementar estrategias pedagógicas para apoyar a los estudiantes que presenten (...) bajos logros o desempeńos superiores en su proceso formativo.

- Determinar la promoción de estudiantes (...) entre grados y niveles de la educación.

- Aportar información para el ajuste e implementación de los planes de mejoramiento (...) de programas académicos.

Los aportes tanto del Ministerio de Educación como de UNESCO, da la posibilidad de interpretar que la evaluación es participativa y que además de focalizar las características y los logros de los estudiantes, debe analizar el currículo y el contexto en el que se implementa y el de desempeño cotidiano de ellos. 
Beneficios de la evaluación psicopedagógica en la institución educativa:

- Proporciona información acerca de la efectividad de los procesos en la gestión para que los estudiantes aprendan; por ejemplo, permite a los consejos directivos planificar cómo administrar sus recursos para apoyarlos, favoreciendo el impulso de prácticas innovadoras, y gestionar el tipo de formación que requieren sus maestros.

- Para los docentes, la evaluación permite conocer el nivel actual de logro de un estudiante y como él o ella aprenden mejor, lo cual permite al maestro planificar qué enseñar y cómo enseñarlo de manera efectiva.

- La evaluación puede entregar información detallada acerca de cómo aprenden y cómo responden los estudiantes a ciertos estilos de enseñanza. Llegando en ocasiones en constituirse en la base para planificar los cambios en la enseñanza. La intención radica en que la evaluación se aplique de manera frecuente y rigurosa y conduzca a un proceso de mejoramiento continuo.

- La evaluación podría enfocarse más en los procesos pedagógicos que orientan los docentes y en la dinámica del el aula, de manera que se analice la efectividad de la enseñanza en función del aprendizaje del estudiante. Implica reflexionar sobre qué permite el logro en el estudiante, qué dificultades se observan, a qué responden éstas y como mejorar la práctica.

La técnica de observación de apoyo en la implementación de la evaluación psicopedagógica:

- El proceso de observación es una técnica que todos los docentes realizan para registrar el proceso en el aula, el aporte en el desarrollo de ésta técnica, posibilita la reflexión y la toma de decisiones en la gestión pedagógica, de lo que sucede en el contexto. Esto significa que es necesario en la formación inicial y en el ejercicio de la profesión, se proporcionen los elementos conceptúales y procedimentales para que se le de el uso a ésta técnica con fines pedagógicos.

- La observación se puede estructurar y focalizar mediante listados de cotejo o comentarios del logro del estudiante. También es posible aumentar su utilidad si se realiza con cierta regularidad y los registros de observación se archivan junto con ejemplos del trabajo de los estudiantes. Así se construye un perfil acumulativo del desarrollo de su proceso. Esto facilita la identificación de nuevos logros o patrones de dificultades recurrentes. También significa que el docente puede trascender el informe del estudiante mucho más detallado que un simple registro de notas. Lo importante es que el maestro tenga claro, qué intenta aclarar y que sobre la base de los resultados, pueda desarrollar un programa de enseñanza.

- La observación como herramienta en la evaluación genera que el docente potencie su habilidad en la práctica cotidiana, posibilitando:

$\checkmark \quad$ La evaluación auténtica: en vez de evaluar una repetición mecánica del aprendizaje, se evalúan actividades de la "vida real"

$\checkmark \quad$ La evaluación conductual: se enfoca en la evaluación de conductas observables y el impacto de factores contextuales en dicha conducta (por ejemplo, qué estudiantes conforman los grupos, la hora del día, las acciones del maestro, entre otros.) 
$\checkmark \quad$ La evaluación de destrezas: divide una tarea compleja en sub-tareas más simples, y evalúa el desempeńo del estudiante en cada una, tal como es enseñada.

$\checkmark$ Evaluación de portafolio: se reúne una selección del trabajo del estudiante que muestra su desarrollo en el tiempo y se le invita a que contribuya con su propia evaluación.

Una propuesta de formación para los docentes bajo un enfoque de educación inclusiva debe enfatizar habilidades para el ejercicio profesional en:

Orientación de la evaluación en competencias básicas de todos los estudiantes, incluyendo aquellos que presentan mayores dificultades. Definición de estrategias pertinentes para evaluar a aquellos estudiantes cuyo logro es bajo y su progreso es lento.

* Aplicación de la evaluación como herramienta de planificación para todos los estudiantes.

* Orientación de la evaluación como apoyo en la toma de decisión en la flexibilidad y adaptación curricular.

* Observación de situaciones de aprendizaje de los estudiantes, incluyendo el uso de listados de cotejo simples y programas de observación.

* Utilización de la información en procesos de interpretación y la toma de decisiones en el currículo y la práctica pedagógica.

* Orientación de procesos y metas de desarrollo en niños y niñas de la educación inicial.

* Implementación de la cooperación como herramienta de trabajo con otros profesionales, de manera que se emplee la evaluación con fines pedagógicos.

La evaluación como apoyo pedagógico al proceso de aprendizaje tiene en cuenta que:
> El proceso de evaluación se focaliza tanto en el estudiante como en el entorno de aprendizaje.

> Quien evalúa debe conocer la propuesta pedagógica de la institución.

> El proceso de evaluación es continuo más que un evento aislado.

> Entre las evaluaciones se incluyen formas de trabajarlas desde el maestro de grado y área, padres de familia y el mismo estudiante.

> El proceso de evaluación incluye una relación entre los aportes de especialistas, maestros, los padres y los estudiantes mismos.

> Los padres de familia son claves al momento de contribuir a la evaluación, ellos aportan una visión y experiencias a las cuales no tienen acceso los maestros ni otros profesionales. Por ejemplo:

- Proveen información acerca del proceso del estudiante fuera de la institución, identifican fortalezas y dificultades no aparentes en el contexto escolar.

- Describen el desarrollo del estudiante durante su primera infancia.

- Orientan a establecer objetivos y hacer programas de trabajo que reconozcan como "propios" y, en consecuencia, apoyarán con mayor seguridad.

- Realimentan de manera rápida a los maestros y especialistas sobre la efectividad de su trabajo con el estudiante.

De manera implícita la evaluación desde la postura de la psicopedagogía va de la mano con el aprendizaje significativo, en función del desarrollo del currículo; en éste se reconocen dos tendencias, de un lado las adaptaciones y de otro la flexibilidad.

La flexibilidad lleva inherente el abanico de posibilidades desde el currículo, piensa en el 
aprendizaje de todos los estudiantes, prepara el contexto, se diversifica cuando es necesario y el maestro está en disposición y formado para atender la necesidad educativa cuando se manifiesta, agregando que su orientación es una responsabilidad de la comunidad educativa.

La adaptación es una forma de abordar la individualización didáctica que permite cambios (que pueden ir desde leves modificaciones en los instrumentos de evaluación hasta la eliminación de contenidos y/o objetivos) en los elementos del currículo (evaluación, metodología, estándares, competencias y objetivos) con el objetivo de optimizar los procesos de enseñanza/ aprendizaje para uno o varios estudiantes y definir aprendizajes básicos y de funcionalidad para el estudiante.

Desde el enfoque de educación inclusiva, la institución toma decisiones con relación a la flexibilización del currículo y pasa a considerar las adaptaciones necesarias que no fueron resueltas en el proceso de flexibilidad. Precisando cómo puede aprender, de qué es capaz, cómo y con qué ayudas; se hace necesario contemplar en este proceso la evaluación individual, inmersa en un currículo flexible, significa que cada estudiante tiene la propuesta de objetivos, metas adecuadas, aprendizajes básicos y niveles de desempeño a sus posibilidades y necesidades, además determina los apoyos y recursos para el desarrollo y movilización del aprendizaje.

La evaluación bajo la mirada de determinar situaciones de los contextos, permite cualificar procesos pedagógicos y deja de tener la connotación de valorar un resultado e impacta en la práctica profesional e institucional y en el desarrollo del currículo. Posibilita que el estudiante independiente de sus condiciones permanezca y se promocione en el servicio educativo, a partir de sus fortalezas. 
Tdea Jorge Ivan Correa Alzate

\section{Bibliografía}

Arnaiz, P. S. (1999). Desafíos de la Especial en el umbral del siglo XXI. Diversidad y educación (pág. 18). Murcia: Universidad de Murcia.

Colombia, M. d. (2009). decreto 1290. Bogotá D.C.: MEN .

Correa, A. I. (2001). Congreso Internacional de Atención a la Diversidad. Evaluación e Intervención Psicopedagógica (pág. 14). Saltillo: SEM.

López, M. J. (s.f.). Psicopedagogía . Recuperado el 23 de Enero de 2005, de La

Evaluación Psicopedagógica: http://www. psicopedagogia.com/

UNESCO. (2004). Temario Abierto sobre Educación Inclusiva. Chile : UNESCO. 